\title{
Use of Video Goggles to Distract Patients During PET/CT Studies of School-Aged Children
}

\author{
Michael J. Gelfand, Jennifer M. Harris, CNMT, Amanda C. Rich, CCLS, and Chelsea S. Kist, CNMT \\ Section of Nuclear Medicine, Department of Radiology, Cincinnati Children's Hospital Medical Center, Cincinnati, Ohio
}

This study was designed to evaluate the effectiveness of video goggles in distracting children undergoing PET/CT and to determine whether the goggles create CT and PET artifacts. Methods: Video goggles with small amounts of internal radioopaque material were used. During whole-body PET/CT imaging, 30 nonsedated patients aged 4-13 y watched videos of their choice using the goggles. Fifteen of the PET/CT studies were performed on a scanner installed in 2006, and the other 15 were performed on a scanner installed in 2013. The fused scans were reviewed for evidence of head movement, and the individual PET and CT scans of the head were reviewed for the presence and severity of streak artifact. The CT exposure settings were recorded for each scan at the anatomic level at which the goggles were worn. Results: Only one of the 30 scans had evidence of significant head motion. Two of the 30 had minor coregistration problems due to motion, and 27 of the 30 had very good to excellent coregistration. For the 2006 scanner, 2 of the 14 evaluable localization CT scans of the head demonstrated no streak artifact in brain tissue, 6 of the 14 had mild streak artifact in brain tissue, and 6 of the 14 had moderate streak artifact in brain tissue. Mild streak artifact in bone was noted in 2 of the 14 studies. For the 2013 scanner, 7 of 15 studies had mild streak artifact in brain tissue and 8 of 15 had no streak artifact in brain tissue, whereas none of the 15 had streak artifact in bone. There were no artifacts attributable to the goggles on the ${ }^{18} \mathrm{~F}-\mathrm{FDG}$ PET brain images of any of the 29 evaluable studies. The average CT exposure parameters at the level of the orbits were $36 \%$ lower on the 2013 scanner than on the 2006 scanner. Conclusion: Video goggles may be used successfully to distract children undergoing PET with localization CT. The goggles cause no significant degradation of the PET brain images or the CT skull images. The degree of artifact on brain tissue images varies from none to moderate and depends on the CT equipment used.

Key Words: patient distraction; video; PET/CT

J Nucl Med Technol 2016; 44:227-229

DOI: 10.2967/jnmt.116.179473

\footnotetext{
Received Jun. 8, 2016; revision accepted Aug. 8, 2016.

For correspondence contact: Michael J. Gelfand, Department of Radiology, Cincinnati Children's Hospital Medical Center, 3333 Burnet Ave., Cincinnati, OH 45229.

E-mail: michael.gelfand@cchmc.org

Published online Sep. 15, 2016.

COPYRIGHT (c) 2016 by the Society of Nuclear Medicine and Molecular Imaging, Inc.
}

$\mathbf{H}$ igh-quality pediatric nuclear medicine imaging, including PET, PET/CT, SPECT, SPECT/CT, and planar single-photon imaging, requires that a child be still during the acquisition. For hybrid imaging, notably PET/CT and SPECT/CT, the patient must remain still not only to prevent degradation of the 3-dimensional PET or SPECT images but also to maintain precise coregistration between the 3-dimensional scintigraphic images and the localization CT images that are acquired after them. Some children may become uncooperative because of fear or anxiety, and others may simply have difficulty remaining still for an acquisition that may be as long as $20-40 \mathrm{~min}$ (1). As a method of distracting patients from the imaging process, video goggles have successfully been used during MR imaging (2-4). Video goggles provide both a visual and an audio distraction to the child. An age-appropriate movie of the patient's choice can focus the patient's attention during imaging, resulting in better cooperation and making it easier for the patient to remain still.

Video goggles contain minimal metal. The amount may be so small that it is possible to acquire head images during PET/CT that do not contain significant artifacts. The purpose of this study was to evaluate the use of video goggles for distracting children during whole-body PET/CT and to evaluate the frequency and severity of CT and PET artifacts caused by the goggles.

\section{MATERIALS AND METHODS}

A video goggle set containing minimal internal radioopaque material was identified and used in this study (1200DX; Vuzix). A child life specialist provided developmentally appropriate sensory preparation for patients before imaging to lessen any fear or anxiety, ensure that each child understood what he or she would experience, and highlight the importance of remaining still. Thirty patients, aged 4-13 y (median age, $9 \mathrm{y}$; mean, $8.8 \mathrm{y}$ ), watched videos of their choice using the goggles while undergoing ${ }^{18} \mathrm{~F}-$ FDG PET/CT, and the images were reviewed. Studies were excluded if the video goggles had slipped out of position from directly in front of the eyes. Fifteen studies were performed on each of two PET/CT scanners, one that had been installed in 2006 (DSTe; GE Healthcare) and one that had been installed in 2013 (Ingenuity; Philips). PET and localization CT axial sections were reviewed individually and as fused images. Streaks or other artifacts caused by the video goggles were noted and evaluated for severity. The CT exposure settings were recorded for each scan at 
FIGURE 1. 7-y-old child imaged on 2006 PET/CT scanner. (A) Image showing moderate soft-tissue streak artifact when viewed at soft-tissue window (120 kVp and $25 \mathrm{mAs}$ ). (B) Adjacent axial CT section from same study showing very mild bone artifact when viewed at bone window. (C) PET image at same level as in B showing no artifact.

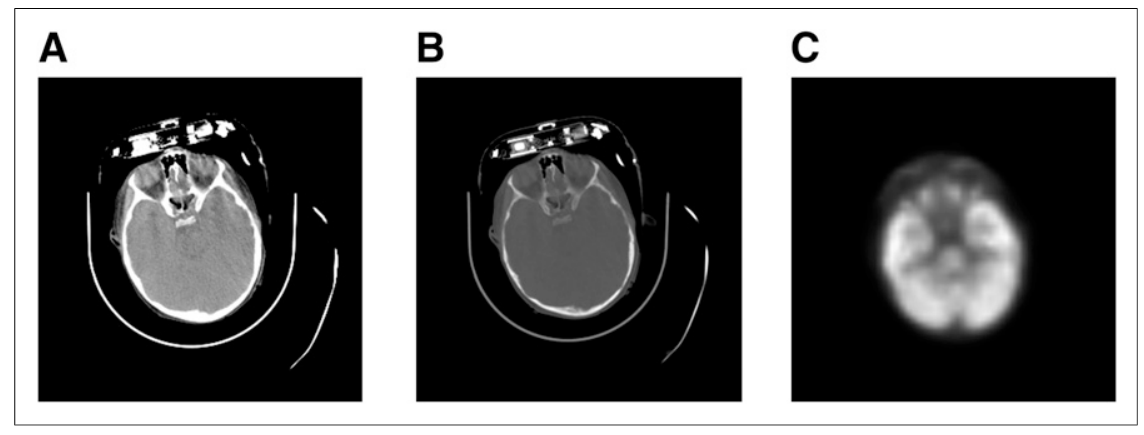

the axial level at which the goggles were worn. The images were also reviewed for evidence of patient motion, including inaccurate coregistration.

The study was a retrospective review of clinical data and was approved by the Institutional Review Board at our hospital, where the patients were imaged. The need to obtain informed consent was waived by the Institutional Review Board.

\section{RESULTS}

All 30 examinations were completed.

\section{Accuracy of PET and CT Coregistration}

Of the 30 scans, only one showed significant motion of the head. This motion precluded accurate coregistration of the PET and CT scans at the level of the head and neck. Two of the 30 scans showed minor coregistration problems due to motion, and 27 of the 30 demonstrated very good to excellent coregistration. The scan with the significant motion was excluded, leaving 29 scans to be further evaluated.

\section{Streak Artifact}

Fourteen of the 15 studies imaged on the 2006 scanner could be evaluated. Two of the 14 localization CT scans of the head demonstrated no streak artifact in brain tissue (viewed at soft-tissue windows), 6 of the 14 had mild streak artifact in brain tissue, and 6 of the 14 had moderate streak artifact in brain tissue. Mild streak artifact in bone was noted in 2 of the 14 studies (viewed at bone windows).

For the 2013 scanner, 7 of the 15 localization CT studies had mild streak artifact in brain tissue and 8 of the 15 had no streak artifact in brain tissue, whereas none of the 15 studies had streak artifact in bone.
There were no artifacts attributable to the goggles on the ${ }^{18}$ F-FDG PET brain images of any of the 29 evaluable studies (Figs. 1 and 2).

On the older scanner, the axial sections were acquired at $120 \mathrm{kVp}$ and an average $\mathrm{mAs}$ of 56 . On the newer scanner, the scans of 12 patients were acquired at $100 \mathrm{kVp}$ and an average $\mathrm{mAs}$ of 30 , and the scans of the remaining 3 patients were acquired at $120 \mathrm{kVp}$ and an average $\mathrm{mAs}$ of 21 . Taking into account peak kilovoltage, milliampere-seconds, and differences in pitch, the average CT exposure parameters at the level of the orbits were calculated to be $36 \%$ lower for the 2013 scanner than for the 2006 scanner.

\section{DISCUSSION}

Video goggles were used successfully as a distraction tool for children undergoing PET/CT. Providing the patient with an opportunity to choose and watch a preferred movie during imaging encourages and assists them in remaining still, as they can focus their attention on the movie. In only 1 of 30 imaging examinations was there significant misregistration between the PET and CT images.

In research studies that have used video goggles during MRI, the distraction effect was greatest in children between 3 and $4 \mathrm{y}$ old and $10 \mathrm{y}$ old. In one study, the benefit of using video goggles during MRI was considered to be $84 \%$. In other studies, the need for sedation was reduced from $53 \%$ to $40 \%(2-4)$. The average estimated cost of sedation at 5 U.S. children's hospitals in 2014 was $\$ 2,950$. Medication for sedation may affect respiratory drive, airway patency, and protective airway reflexes, and appropriately trained

FIGURE 2. 11-y-old child imaged on 2013 PET/CT scanner. (A) Image showing minimal soft-tissue streak artifact when viewed at soft-tissue window (100 kVp and $13 \mathrm{mAs}$ ). (B) Same axial section as in $A$ showing no bone artifact when viewed at bone window. (C) PET image at same level as in B showing no artifact.

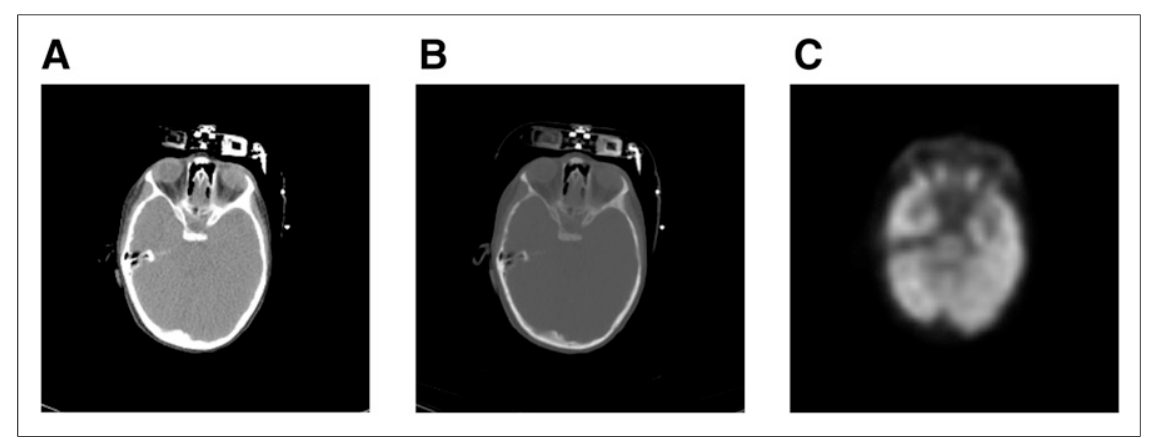


personnel, special equipment, and monitoring are required (5). One study found that when sedation was avoided, the time required to prepare and observe patients was reduced by $2 \mathrm{~h}(6)$.

There were no visible artifacts on axial PET images of the brain, although artifacts were sometimes seen on the axial localization CT images. The degree of artifact in brain tissue on axial localization CT images varied from none to moderate and depended on the CT equipment and exposure parameters used. Streak artifact in the brain parenchyma was less common on CT images acquired with the newermodel CT scanner despite use of considerably lower exposure parameters. Other measurements comparing CT dose from these two scanners indicated an $88 \%$ reduction in radiation dose for the entire scan (7).

\section{CONCLUSION}

The use of video goggles as a patient distraction device during PET scans performed with localization CT did not significantly degrade the axial PET images of the brain. The axial CT images of the brain and skull were adequate for localization and attenuation correction. The degree of artifact on axial images of brain parenchyma and bone varied from none to moderate and depended on the CT equipment and exposure parameters used.

\section{DISCLOSURE}

No potential conflict of interest relevant to this article was reported.

\section{REFERENCES}

1. Alexander M. Managing patient stress in pediatric radiology. Radiol Technol. 2012;83:549-560.

2. Harned RK II, Strain JD. MRI-compatible audio/visual system: impact on pediatric sedation. Pediatr Radiol. 2001;31:247-250.

3. Lemaire C, Moran GR, Swan H. Impact of audio/visual systems on pediatric sedation in magnetic resonance imaging. J Magn Reson Imaging. 2009;30:649-655.

4. Koch BL. Avoiding sedation in pediatric radiology. Pediatr Radiol. 2008;38 (suppl):S225-S226.

5. Coté CJ, Wilson S, American Academy of Pediatrics, American Academy of Pediatric Dentistry. Guidelines for monitoring and management of pediatric patients before, during, and after sedation for diagnostic and therapeutic procedures: update 2016. Pediatrics. 2016;138:e20161212.

6. Technical research report to the Child Life Council: the economic value of a child life program for non-sedated MR imaging. https://www.childlife.org/files/MR\% 20Nonsedate\%20Tech\%20Report\%20May\%202014.pdf. Published May 15, 2014. Accessed September 1, 2016.

7. Gelfand MJ, Lemen LC, Trout AT, Iverson TM, Sharp SE, Qi Z. CT dose reduction in pediatric and young adult patients through use of a new generation PET/CT scanner [abstract]. J Nucl Med. 2014;55(suppl 1):367. 